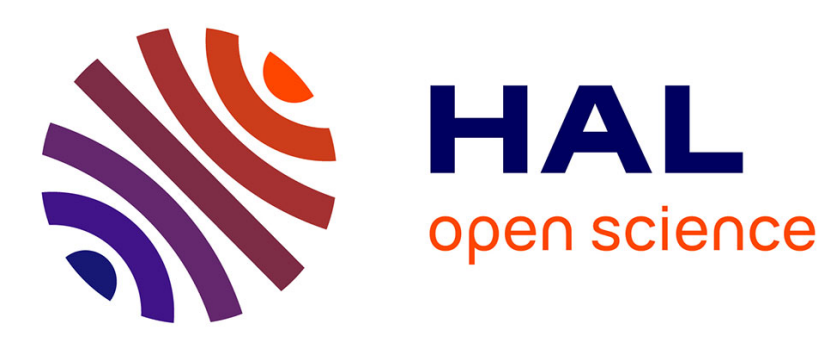

\title{
Equal Cost Multiple Path Energy-Aware Routing in Carrier-Ethernet Networks with Bundled Links
} Rihab Maaloul, Raouia Taktak, Lamia Chaari, Bernard Cousin

\section{To cite this version:}

Rihab Maaloul, Raouia Taktak, Lamia Chaari, Bernard Cousin. Equal Cost Multiple Path EnergyAware Routing in Carrier-Ethernet Networks with Bundled Links. 14th ACS/IEEE International Conference on Computer Systems and Applications (AICCSA 2017), Oct 2017, Hammamet, Tunisia. hal-01660934

\section{HAL Id: hal-01660934 https://hal.science/hal-01660934}

Submitted on 11 Dec 2017

HAL is a multi-disciplinary open access archive for the deposit and dissemination of scientific research documents, whether they are published or not. The documents may come from teaching and research institutions in France or abroad, or from public or private research centers.
L'archive ouverte pluridisciplinaire HAL, est destinée au dépôt et à la diffusion de documents scientifiques de niveau recherche, publiés ou non, émanant des établissements d'enseignement et de recherche français ou étrangers, des laboratoires publics ou privés. 


\title{
Equal Cost Multiple Path Energy-Aware Routing in Carrier-Ethernet Networks with Bundled Links
}

\author{
Rihab Maaloul*, Raouia Taktak* Lamia Chaari*and Bernard Cousin ${ }^{\dagger}$ \\ * Laboratory of Technology and Smart Systems (LT2S), \\ Digital Research Center of SFAX (CRNS), university of Sfax, Tunisia \\ ${ }^{\dagger}$ University of Rennes 1, IRISA, France
}

\begin{abstract}
The reduction of operational expenditure has become a major concern for telecommunication operators and Internet service providers. In this paper, we propose an energy aware routing (EAR) in Carrier Ethernet networks operating with Shortest Path Bridging (SPB) protocol with equal cost multi-path (ECMP). Since traffic load has no influence on power consumption of Carrier Ethernet network elements, the conventional solution to reduce power consumption is to find the maximal set of network elements that can be turned off/on so that the network performance is not deteriorated. To tackle this optimization problem, we propose an exact method based on Mixed Integer Linear Programming (MILP) formulation, called SPB energy-aware routing (SPB-EAR). Since SPB-EAR is proved to be NP-hard, we present two heuristics algorithm suitable for large-sized networks, called Green SPB (G-SPB) and Fast Greedy SPB (FG-SPB). In this work, we consider that a connection between two nodes is represented by bundled link consisting of multiple cables. Experimentations on four realistic network topologies show that G-SPB and FG-SPB can save almost as much power consumption as SPB-EAR.
\end{abstract}

Keywords-Energy-Aware Routing; Green Network; Carrier Ethernet; SPB Protocol; Network Optimization; Bundled Link

\section{INTRODUCTION}

The reduction of energy expenditure has become a major concern for telecommunication operators and Internet service Providers (ISP). In recent years, network providers has considered Carrier Ethernet as an effective inter-connection technology for metro and core network segments. In fact, Carrier Ethernet extends the original set of Ethernet LAN technologies by supporting further capabilities to meet the requirements of next generation broadband networks. For instance, $100 \mathrm{Gbit} / \mathrm{s}$ Ethernet is becoming the key enabler for more cost-effective backbone Internet Service Provider (ISP) networks [1] [2]. Moreover, it is massively used to deliver data center applications [3] [4]. The energy consumption of Carrier Ethernet devices is largely dependent of their installed capacity [5] [6]. By its characteristic, the energy consumed by a switch increases linearly with the number of linecards plugged into the switch as well as the number of active ports on each card [7]. For these kind of devices, two basic techniques can be adopted to reduce the power consumption. The first one, is the so-called power scaling or Adaptive Link Rate (ALR), which allows dynamic modulation of the device capacity to be proportional to the traffic load. The second one, named energy-aware routing (EAR), forces some selected devices (e.g. the least used devices) to be turned off and consolidating the traffic on a small set of network devices. Current network devices cannot support ALR technique, thus architectural designs of network elements should be undertaken. However, efficient traffic engineering can achieve an EAR by smartly aggregating traffic demands into a small number of network elements and turning off unnecessary elements. In this paper, we focus on saving energy by applying EAR algorithm to Carrier Ethernet networks.

The routing protocol considered in this work is the SPB (Shortest Path Bridging) standardized by IEEE 802.1aq [8]. SPB computes the shortest path between any pair of nodes. Indeed, it aims to ensure frame forwarding on the shortest path within a Shortest Path Tree (SPT) spanning the network by using an extension of the Intermediate System to Intermediate System (IS-IS) link state routing protocol [9]. IS-IS protocol supports the handling of MAC addresses; it is able to run directly over Ethernet as it is not tight to IP. In order to guarantee the load balancing requirement, SPB allows the use of ECMP-based routing strategy [10]. ECMP improves network bandwidth utilization, allows an enhanced use of mesh topologies, and provides additional resiliency by enabling fast access to backup paths. Moreover, the concept of bundled link could help to favor the network connectivity along with improved resilience in case of a link failure. This technique of bundled link, called also link aggregation, is standardized by the IEEE 802.1AX [11] and revised in [12]. This standard defines the bundled link capability (which is MAC independent), and general information relevant to specific MAC types that support bundled link. The two main reasons behind using bundled links are (i) allowing network operators to easily upgrade their network capacity, (ii) resilience and network stability in case of cable failure and congestion. In this paper, the term 'link' refers to one bundled link that is composed by multiple cables.

In order to model an EAR compliant with SPB protocol, we make use of optimization techniques. Our main contributions are:

- First, we formulate a MILP, called SPB-EAR, based on the MILP given in [13],whose objective is to solve 
the problem of reducing power consumption in SPBbased Carrier Ethernet networks. To the best of our knowledge this is the first work that jointly minimizes the number of active cables in bundled links whilst satisfying ECMP routing rule.

- Second, we propose two heuristics, called G-SPB (Green SPB) and FG-SPB (Fast Greedy SPB), to solve SPB-EAR. The main difference between the two heuristics is the selection order of candidate bundled links to power-off.

The paper is organized as follows. We present related works in the next Section. In Section III, we formally describe the problem and model it as an MILP formulation. In Section IV, we describe our related heuristic algorithms. A performance analysis of the proposed resolution methods is presented in Section V. Finally, Section VI is devoted to giving concluding remarks and new directions for future work.

\section{RELATED WORKS}

Related to literature addressing EAR, we found few researches focus on energy saving in Carrier Ethernet networks [14] [15]. Moreover, none of them cares about saving energy considering SPB protocol. In order to better analyze EAR approach, we choose to study IP approaches which propose EAR protocols compatible with SPB-based Carrier Ethernet context. In this respect, [13] and [16] propose EAR operated with the very popular shortest path protocol, i.e. OSPF, which uses ECMP policy to prevent network congestion. The authors in [13] [17] assume to turn off only the links since turning on/off an entire device may reduce device life cycle. Moreover, the authors in [13] have proposed to use heuristic approach to find a stable OSPF weight setting, along with robust optimization design, for multi-period traffic matrices. As for [17], authors have used genetic algorithms to find the link weights that influence the traffic distribution optimizing both energy-efficiency and load-balancing. Efficient greedy heuristics with different sorting criteria are proposed in [18] and [16], exploiting the possibility to turn off both nodes and links but, in [16], the links weight is optimized so as to reduce both power consumption and network congestion. Fortz et al in [19] have resorted to a local search heuristic by iteratively modifying the OSPF weights setting for load balancing purpose only. Some relevant works have sketched the problem of energyaware traffic engineering with bundled links by turning off single cables. In [20], which was the first work dealing with energy-aware traffic engineering considering bundled links, the authors formulate the problem as an Integer Linear Program (ILP), and propose to power-off single cables. Moreover, the total load is balanced over all links maximizing the residual capacity of links, which can therefore be powered off. Moreover, this solution does not consider any specific routing rule for traffic demands except the classical flow-conservation constraints. In [21], the authors have proposed an energy-aware traffic engineering that aims at maximizing the number of cables to be powered off while respecting the given traffic demands and maintaining a required level of network reliability.

\section{GREEN ECMP ROUTING PROBLEM}

\section{A. Network model and notations}

In this work, we aim at reducing energy consumption in Carrier Networks so we propose to implement EAR on Carrier Ethernet network operated with SPB protocol (SPB-EAR). We show through an example in Figure 1 how SPB-EAR can be applied in this context. We consider the undirected graph $G(V, E)$ with $V=\{0,1,2,3,4,5\}$ and $E=\{(0,1),(0,2),(0,3),(1,4),(2,4),(3,4),(4,5)\}$, each node represent bridge/switch and each link $(u, v)$ represents a bundled link with finite capacity $C_{u v}>0$. We assume that each link is comprised of 3 cables, i.e., with bundle size equals to three, and capacities on links are as depicted in Figure 1a. The capacity of a link is the total capacity of its operational cables. We denote $f_{u v}$ the total flow on link $(u, v) \in E$. Let $D$ be the set of all traffic demands in $G$ and the triple $\left(s_{d}, t_{d}, h^{d}\right)$ refers to the demand indexed by $d=1,2, \ldots,|D|$ between node $s_{d} \in V$ and node $t_{d} \in V$, where $h^{d}$ is the amount of traffic exchanged between $s_{d}$ and $t_{d}$. Let $S P B_{d}=\left\{s p b_{d^{q}} \mid\right.$ all $(s, t)$ shortest paths for traffic demand $d$, indexed by $q>0\}$. Let $D_{T M_{p}}$ denote the traffic matrix that collects all the demands at period $p$. In the example of Figure 1, we assume that there are 2 traffic matrices $D_{T M 1}$ and $D_{T M 2}$ at two different periods. Each traffic matrix has two traffic demands: $D_{T M 1}=$ $\{(0,4,6),(0,5,7)\}$, and $D_{T M 2}=\{(0,4,3),(0,5,4)\}$. For $D_{T M 1}$, the traffic demands are equally split over 3 different paths from node 0 to node 4. i.e., $S P B_{1}=$ $\left\{s p b_{1^{1}}=(0,2,4), s p b_{1^{2}}=(0,1,4), s p b_{1^{3}}=(0,3,4)\right\}$, $S P B_{2}=\left\{s p b_{2^{1}}=(0,2,4,5), s p b_{2^{2}}=(0,1,4,5), s p b_{2^{3}}=\right.$ $(0,3,4,5)\}$. We obtain $f_{01}=f_{02}=f_{03}=f_{14}=f_{24}=$ $f_{34}=(6+7) / 3=4.33<5$. This routing is feasible but there is no possible way to turn off any link, we can only remove one cable from the link $(4,5)$ since $f_{45}=$ $7<13(2 / 3)=8.6$. However, in the case of $D_{T M 2}$ (where the demand ends are identical to $D_{T M 1}$ but the demand capacities are lower) more links can be powered off. Indeed the decreasing traffic demands may be equally split among 2 different paths between node 0 to node 4 . i.e., $f_{01}=f_{03}=$ $f_{14}=f_{34}=(3+4) / 2=3.5<5$. So, we can totally turn off the two links $(0,2),(2,4)$, besides, turning off 2 cables from the link $(4,5)$, i.e, $f_{45}=4<13 *(1 / 3)=4.33$. For the first traffic matrix, only $4.76 \%$, i.e., $(1-(20 / 21)) \times 100$ power consumption can be saved, while for the second traffic matrix $33.33 \%$, i.e., $(1-(13 / 21)) \times 100$ of power saving can be reached. The power saving computation is described in Section V. 


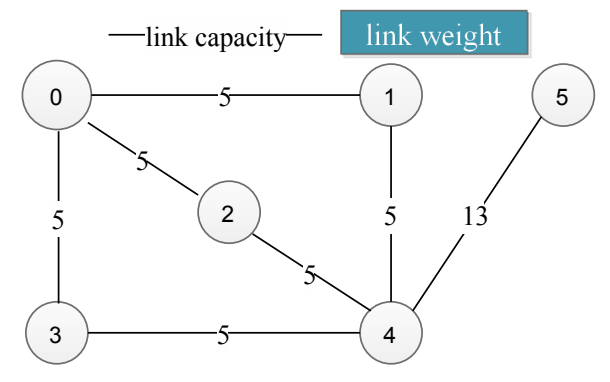

(a) Network topology before using EAR

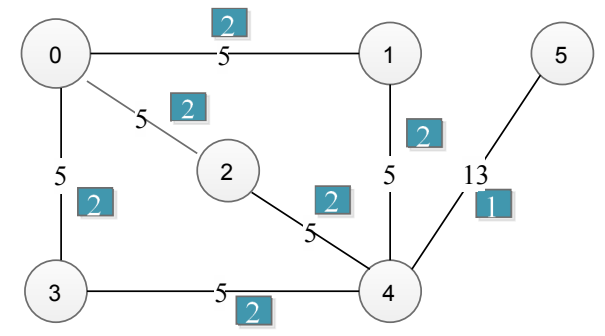

(b) Network topology using EAR for $D_{T M 1}$

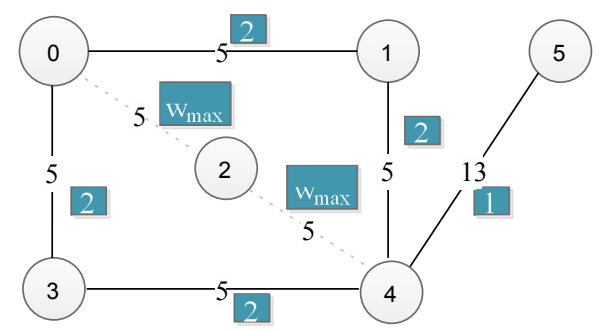

(c) Network topology using EAR for $D_{T M 2}$

Figure 1: Example of network topology for EAR

Energy-aware traffic engineering allows to assign an appropriate links weight setting for each traffic matrix independently. The links weight is used to compute the shortest path (the sequence of links used by a demand) and a link can be powered off by assigning a very large value to its weight and therefore it could be excluded from the shortest paths. The problem of ECMP weight setting is known to be NPhard [16], [17], [19]. In this paper we assume that we use any solution provided by any of the existing solutions (for instance one provided by [13]). For the sake of simplicity and without loss of generality, in heuristic algorithms we consider that the initial weight setting uses the inverse of link capacity. If the bundled link $e$ is still used in the new routing solution its weight remains stable. Otherwise, its weight is changed to $w_{\max }$.

\section{B. Problem Formulation}

In this section, we propose a MILP programming formulation for the SPB-EAR problem. Table I summarizes notations and parameters of the model.
Table I: Summary of notations and parameters

\begin{tabular}{|c|c|}
\hline Parameters & Description \\
\hline $\mathrm{G}=(V, E)$ & $\begin{array}{l}\text { Undirected graph where } V \text { is the set of vertices (nodes) and } E \text { is the } \\
\text { set of edges (links) }\end{array}$ \\
\hline$E^{\prime}$ & Set of links used to route traffic \\
\hline $\mathcal{E}_{u v}$ & Power consumption of a powred cable in link $(u, v) \in E$ \\
\hline$\beta$ & $\begin{array}{l}\text { Parameter set to } 0.1 \text {, assuming that the powered-off cables consume } \\
10 \% \text { of the power spent in the active mode }\end{array}$ \\
\hline$C_{u v}$ & Capacity of link $(u, v) \in E$ \\
\hline$\mu$ & Maximum tolerated link utilization; $\mu \in] 0,1]$ \\
\hline$N_{G}(u)$ & Set of neighbors of $u \in V$ \\
\hline$D$ & Set of all traffic demands $D=\left\{\left(s_{d}, t_{d}, h^{d}\right), s_{d} \in V, t_{d} \in V\right\}$ \\
\hline$D^{t}$ & Set of all destination nodes $t \in V$ \\
\hline$h^{d}$ & Demand of the traffic flow from node $s_{d}$ to $t_{d}$ \\
\hline$B_{e}$ & Bundle size of link $e \in E$ \\
\hline$n_{u v}$ & $\begin{array}{l}\text { Integer variable to indicate the number of powered-on cables in link } \\
(u, v)\end{array}$ \\
\hline$x_{u v}$ & $\begin{array}{l}\text { Binary variable to indicate if the link }(u, v) \text { has at least one powered- } \\
\text { on cable or not }\end{array}$ \\
\hline$f_{u v}^{d}$ & $\begin{array}{l}\text { Real variable to present the amount of flow of the demand } d \text { that is } \\
\text { routed traversing the link }(u, v) ; f_{u v}^{d} \in\left[\begin{array}{ll}0 & 1\end{array}\right]\end{array}$ \\
\hline$f_{u v}$ & $\begin{array}{l}\text { Real variable to present the total flow traversing the link }(u, v) ; f_{u v} \geq \\
0\end{array}$ \\
\hline$r_{u v}^{t}$ & $\begin{array}{l}\text { Binary variable to determine whether link }(u, v) \\
\text { belongs to one of shortest paths from } u \text { to } t \text { (i.e., using ECMP) }\end{array}$ \\
\hline$z_{u}^{d}$ & $\begin{array}{l}\text { Real variable to represent fraction of the demand } d \text { routed on the } \\
\text { outgoing node } u \text { belonging to one of shortest paths from } s \text { to } t \text { (i.e., } \\
\text { using ECMP); } z_{u}^{s t} \in\left[\begin{array}{ll}0 & 1\end{array}\right]\end{array}$ \\
\hline$k_{u}^{t}$ & Real variable to present the cost of shortest path from $u$ to $t$ \\
\hline $\mathrm{M}$ & Non-negative and a big enough constant \\
\hline$w_{\max }$ & $\begin{array}{l}\text { Maximum value of link weight assigned to the powered-off link (i.e., } \\
\text { all its cables are powered-off) }\end{array}$ \\
\hline$w_{u v}$ & Weight of the link $(u, v) \in E ; 1 \leq w_{u v} \leq w_{\max }$ \\
\hline
\end{tabular}

$$
\min \sum_{e \in E} n_{e} \mathcal{E}_{e}+\beta\left\{\sum_{(e) \in E}\left(B_{e}-n_{e}\right) \mathcal{E}_{e}\right\}
$$

$$
\sum_{v \in N_{G}(u)}\left(f_{v u}^{d}-f_{u v}^{d}\right)=\left\{\begin{array}{lll}
-1 & \text { if } u=s_{d}, \\
1 & \text { if } u=t_{d}, & \forall u \in V \\
0 & \text { if } \mathbf{u} \neq s_{d}, t_{d}, & d \in D,
\end{array}\right.
$$

$$
\begin{gathered}
f_{u v}=\sum_{d \in D} h^{d}\left(f_{u v}^{d}+f_{v u}^{d}\right) \leq \mu\left(n_{e} / B_{e}\right) C_{e} \\
\forall e=(u, v) \in E, \\
x_{e} \leq n_{e} \quad \forall e \in E, \\
B_{e} x_{e} \geq n_{e} \quad \forall e \in E, \\
0 \leq z_{u}^{d}-f_{u v}^{d} \leq 1-r_{u v}^{t} \quad \forall d \in D ;(u, v) \in E, \\
f_{u v}^{d}-r_{u v}^{t} \leq 0 \quad \forall(u, v) \in E ; d \in D, \\
r_{u v}^{t} \leq x_{u v} \quad \forall(u, v) \in E ; t \in V, \\
1-r_{u v}^{t} \leq k_{v}^{t}+w_{u v}-k_{u}^{t} \leq M\left(1-r_{u v}^{t}\right) \\
\quad \forall u, t \in V ;(u, v) \in E, \\
w_{\max }\left(1-x_{u v}\right) \leq w_{u v} \quad \forall(u, v) \in E, \\
w_{u v}+x_{u v} \leq w_{\max } \quad \forall(u, v) \in E, \\
0 \leq n_{e} \leq B_{e} \quad \forall e \in E .
\end{gathered}
$$


The objective function (1) minimizes the total power consumption induced by cables. It is composed of two parts. The first part computes the power consumption of poweredon cables. The second part computes the consumption of powered-off cables. It is weighted by the parameter $\beta$ that is set to 0.1 , assuming that the powered-off cables consume $10 \%$ of the power spent in the active mode. Constraints (2) express the classical flow conservation. They ensure that incoming and outgoing flows are equal for each node except the demand end nodes. Constraints (3) say that the sum of traffic of all demands routed on the link $e=(u, v)$ must not exceed the tolerated link capacity $\mu C_{e}$. We consider that the capacity of a link is shared between the traffic in both directions [22]. Indeed, this model allows to reduce the number of variables without loss of generality. Inequalities (4) make sure that if the link $e$ has no powered-on any cables, then $x_{e}=0$. Inequalities (5) make sure if the link $e$ has at least one cable powered-on (i.e., $n_{e} \geq 1$ ) then $x_{e}=1$. Inequalities (6) are for ECMP routing configuration. They guarantee that if the link $(u, v)$ belongs to one of the shortest path from $u$ to $t$ (i.e., $r_{u v}^{t}=1$ ), then the flow $f_{u v}^{s t}$ is equal to $z_{u}^{s t}$. This latter represents the common value of the flow assigned to all links outgoing from $u$ belonging to the shortest paths from $u$ to $t$. Inequalities (7) force $f_{u v}^{s t}=0$ for all links $(u, v)$ that do not belong to the shortest path from $u$ to $t$. Inequalities (8) forbid powered-off links to belong to one of the shortest paths. Inequalities (9) compute the weight of the link $(u, v)$ congruent with the length of the shortest path from $u$ to $t$. The variable $k_{v}^{t}$ corresponds to the cost/length of the shortest from node to $v$ node $t$. Inequalities (10) and (11) put the weights of powered-off links to $w_{\max }$. Finally, inequalities (12) bound the number of powered-on cables per link to be less or equal to the $B_{e}$.

\section{Heuristic Algorithms}

It is very challenging and sometimes impossible to get an optimal solution in a reasonable time for the previous MILP formulation, mainly for large topologies and dense instances. This is due to the fact that our problem is NPhard. It is indeed a particular case of the problems studied in [20] [13] and proved to be strongly NP-hard. Therefore, to find feasible solutions in reasonable time, we use two greedy heuristics, called Green SPB (G-SPB) and Fast Greedy SPB (FG-SPB). The greedy heuristic has been chosen in our case because it can provide good approximations to the optimum. For the sake of simplicity and without loss of generality, we consider that the initial weight setting uses the inverse of link capacity. Further, the links weight will not be modified only if whole the link is removed, in this case the new link weight will be equal to $w_{\max }$.

\section{A. Green $\operatorname{SPB}(G-S P B)$}

Figure 2 reports a diagram description of the process of G-SPB. It takes into account the network topology
$G=(V, E, W)$ and traffic matrix $D$, the output is a routing solution on $G^{\prime}=\left(V, E^{\prime}, W^{\prime}\right)$, containing only the poweredon cables used to route the demands. G-SPB consists of two main phases. In the first phase, we try to turn off the whole of the bundled link. The intuition considers that the power saving achieved by powering off, initially, the whole link is better than powering off a part of the bundle. We choose to sort links by the amount of traffic already routed through it, the smallest first. In other words, we sort the links in decreasing order of their residual capacities. The heuristic iteratively selects a candidate link to be turned off. At each iteration, a feasible route (SPB performed) is computed. If no feasible route exists, then we put back the selected link in $G^{\prime}$. If no violation of the operational constraints occurs, the selected link is turned off. This process is repeated until no more links can be turned off. The second phase is devoted to turning off as many cables as possible so that all the flow demands are still satisfied. For each used link (i.e., $e \in G^{\prime}$ ) we keep the minimum number of cables by rounding up the following ratio:

$$
n_{e}=\left\lceil\frac{f_{e} B_{e}}{\mu C_{e}}\right\rceil
$$

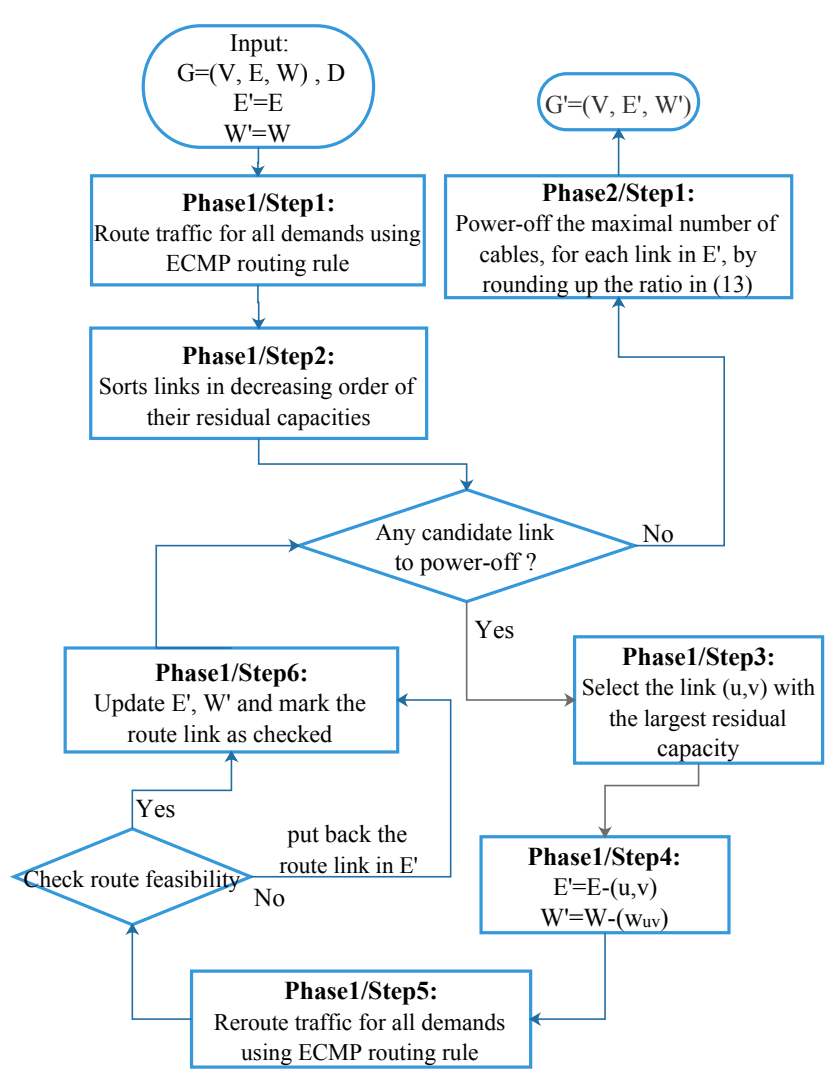

Figure 2: G-SPB diagram

ECMP is conceptually similar to Yen's algorithm [23], that requires $O(K .|V| .(|E|+|V| . \log |V|))$ times to generate 
$K$ shortest paths for each demand. Therefore, G-SPB can be solved in $O(K .|D| \cdot|V| \cdot(|E|+|V| \cdot \log |V|))$.

\section{B. Fast Greedy $S P B$}

Figure 3 reports a diagram description of the process of FG-SPB. The flows in each link take initially the values of the dual variables obtained by solving the MILP (14)(17), that minimizes the total flow summed on each link, subject to the classical constraints of flow conservation and link allowable capacity utilization. This MILP can achieve an upper bound on energy saving for any feasible solution in the case of using at most the sufficient number of cables that satisfies all traffic demands. The work [20] has shown that this solution performs poorly comparing to the optimal one. Therefore, we propose to continue the FG-SPB proceeding as follows. Each unused link will be powered-off, i.e., each link with $f_{e}=0$. The next step sorts the remaining links $E^{\prime}$ in priority with the largest residual capacity. For each candidate link, we try to power-off the maximal number of cables using (13). Then we check the feasibility of SPB route. If it exists, the current link is marked as checked. If the route is not feasible, the cables are powered on and the corresponding link marked as checked. This process is repeated until every link is checked.

The MILP (14)-(17) can take at most $O\left(|E|^{2}\right)$ times. Note that, for the execution time of FG-SPB we exclude the time complexity of the MILP (14)-(17). Thus, FG-SPB (strating from (Phase2/Step1) can be solved exactly in $O(K .|D| \cdot|V| \cdot(|E|+|V| \cdot \log |V|))$.

$$
\begin{aligned}
& \min \sum_{e \in E} f_{e} \\
& \sum_{v \in N_{G}(u)}\left(f_{v u}^{d}-f_{u v}^{d}\right)=\left\{\begin{array}{lll}
-1 & \text { if } \mathbf{u}=s_{d}, \\
1 & \text { if } \mathbf{u}=t_{d}, & \forall u \in V \\
0 & \text { if } \mathbf{u} \neq s_{d}, t_{d}, & d \in D,
\end{array}\right. \\
& f_{e}=\sum_{d \in D} h^{d}\left(f_{u v}^{d}+f_{v u}^{d}\right) \quad \forall e=(u, v) \in E, \\
& f_{e} \leq \mu C_{e} \quad \forall(u, v) \in E .
\end{aligned}
$$

\section{Performance analysis}

In this section, we evaluate the SPB-EAR, and the heuristic-based algorithms (G-SPB and FG-SPB). We start by comparing solutions obtained by the exact formulation (SPB-EAR) with the heuristic ones on the same network instances. Then, we provide a performance analysis of the heuristic solutions for large network instances. We consider realistic network instances collected from SNDlib [24], considering three traffic level (low, medium, high). To evaluate the different traffic load, the traffic matrix is scaled with the load parameter $\gamma$ that is set to three different values $0.5,1$, and 2.5. The performance of the proposed approaches (SPBEAR, G-SPB and FG-SPB) is evaluated using the following metric:

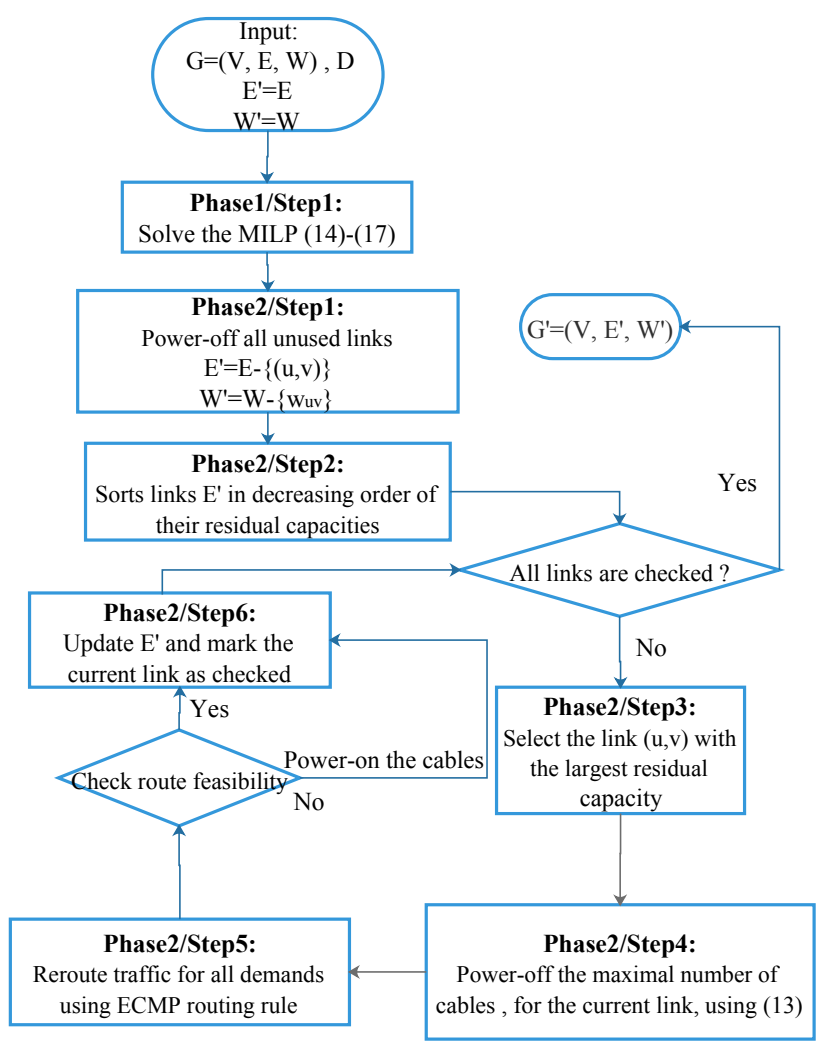

Figure 3: FG-SPB diagram

- $\eta$ indicates a network's power saving that can be obtained. It is computed as follow:

$$
\eta=\left(1-\frac{\sum_{e \in E} n_{e}}{\sum_{e \in E} B_{e}}\right) \times 100 \%
$$

- $\phi$ measures the increase of path cost. In order to report the distribution of this parameter, we calculate for all the demands the difference of costs between an EAR algorithm route and the corresponding ECMP path (before applying any EAR algorithm).

- As load balancing is considered to be a requirement that should be fulfilled in Carrier Ethernet, the third metric is devoted to measuring the fairness of the traffic distribution on the active links E'. The fairness index FI is used to measure whether the traffic load is fairly distributed among all of the links. In our performance analysis, we use Jains Fairness Index [25]:

$$
F I=\frac{\left(\sum_{e \in E^{\prime}} l_{e}\right)^{2}}{\left|E^{\prime}\right| \times \sum_{e \in E^{\prime}} l_{e}^{2}},
$$

where $l_{e}$ is the traffic utilization of the link $e \in E^{\prime}$. Note that, when $F I=1$, this indicates that the traffic 
is distributed in a fair way.

We solved the MILP model using CPLEX 12.6.2 solver with Concert Technology (C++) [26], with a time limit sets to 3 hours (10800 seconds). As known, in practice, network operators do not run their networks at full load in order to avoid transient congestion. In our work, the maximum allowed utilization of links is set to $70 \%(\mu$ $=0.7)$. Both MILP algorithm and heuristics have been tested on four realistic topologies taking into account three different traffic loads. Obtained results are reported in Table II, Table III, and Table IV. Entries of tables are the following. The first column indicates the network instance name. The second column gives the load parameter $\gamma$ by which the traffic matrix is scaled. Energy saving column reports the percentage of powered off cables $\eta$. The gap to the optimum column reports the energy performance of the optimized network, i.e., the ratio (UB-LB)/LB, where $\mathrm{UB}$ is the upper bound on power consumption, the power consumption of the sub-graph solution, and LB is the lower bound on power consumption (the power consumption of the linear relaxation). Note that, the relaxation technique replaces the integer variables of the original MILP by appropriate continuous constraints, Interested readers are referred to [27] for more details. Power $(W)$ is the upper bound on power consumption of the sub-graph solution, i.e., UB. We assume that the power consumption of a single powered-on cable estimated to be $30 \mathrm{~W}$ and the powered-off consumes $10 \%$, i.e., $\beta=0.1$, of the power spent in the active mode. FI reports fairness of traffic distribution. Finally, time column reports the computation time in seconds.

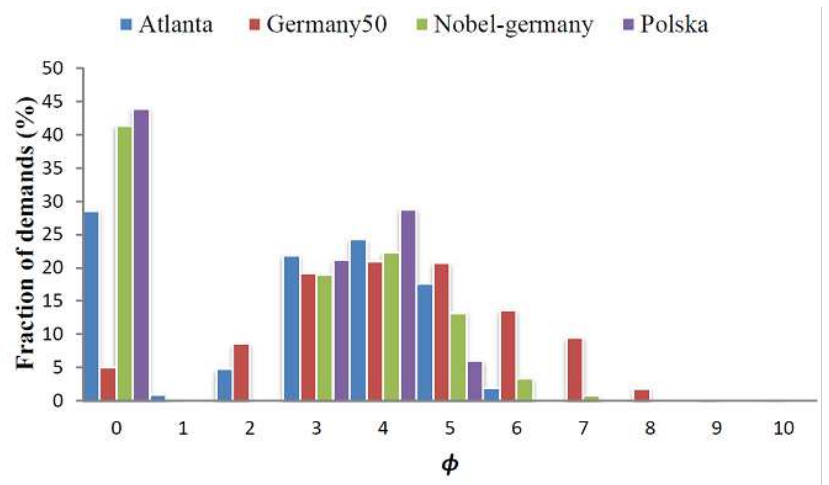

Figure 4: Over-cost induced by G-SPB heuristic

As a first observation, both heuristic algorithms produce encouraging results in terms of execution times. When analyzing the results reported in Table III and Table IV, we can state that G-SPB algorithm performs better than FGSPB. In addition, for Germany50 network G-SPB achieves

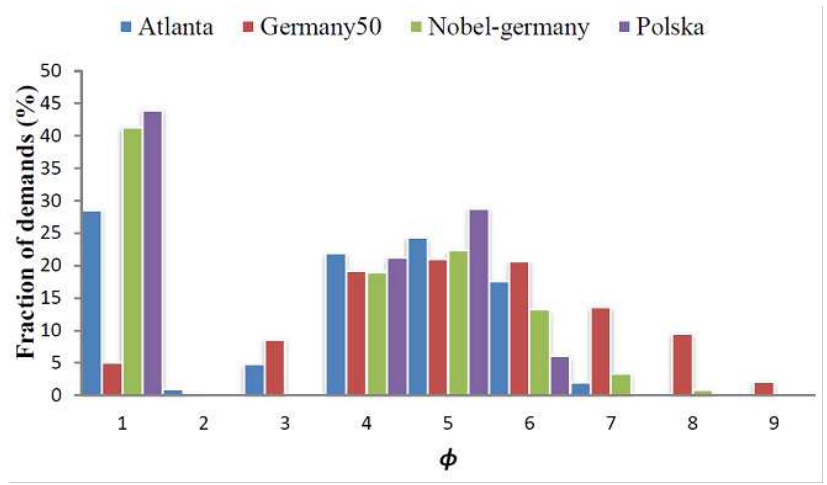

Figure 5: Over-cost induced by FG-SPB heuristic

higher percentage of energy saving compared to the FGSPB heuristic and SPB-EAR algorithm (the MILP model has been stopped before reaching optimality due to large topology and dense instance). It can be noted that, on average, a good load balancing (FI), is obtained by all algorithms for nearly all instances, ranging from 0.43 to 0.79 .

Results clearly show that, as expected, the energy saving decreases when $\gamma$ parameter increases. However, for some instances as the case of Nobel-germany and Polska networks with both heuristics, the percentage of powered off cables remains the same for different values of $\gamma$, i.e., $\gamma=0.5$ and $\gamma=1$. This is obviously due the fact that cables capacities for the latter instances are sufficient to satisfy high traffic demands. Experiments show also that for the execution time for FG-SPB is relatively better than G-SPB. This is obvious because FG-SPB has an initial distribution of traffic provided by the MILP (14)-(17). In summary, compared with MILP solutions, both heuristics perform similarly. Moreover, the solutions provided by G-SPB and FG-SPB especially for large instance (e.g., Germany50) prove the efficiency of our heuristics.

Figure 4 and Figure 5 depict the increase of path cost in terms of over cost caused by both heuristics with respect to SPB routing (the load parameter $\gamma$ is set to 1 ). When the over-cost parameter $\phi$ is equal to 0 , it means that the routing solution is exactly the same the SPB one performed in the original graph. We remark that a significant fraction of demands (from $27 \%$ to $43 \%$ ) is not affected, apart from Germany50 (only about 5\%). However, the path cost can be affected by adding extra cost units to a demand. That is for instance the case of Germany50 network for which $2 \%$ of demands add 9 extra units of cost to their routes. Indeed, the initial link weight setting can impact not only the energy saving but also over-cost paths of the optimized topology.

\section{CONCLUSION}

In this paper, we proposed two heuristics performing energy-aware routing, Green SPB (G-SPB) and Fast Greedy 
Table II: SPB-EAR formulation

\begin{tabular}{|c|c|c|c|c|c|c|c|c|c|}
\hline Network & $|V|$ & $|E|$ & $|D|$ & $\begin{array}{c}\text { load } \\
(\gamma)\end{array}$ & $\begin{array}{c}\text { Saving } \\
(\eta \%)\end{array}$ & $\begin{array}{c}\text { Gap } \\
(\%)\end{array}$ & $\begin{array}{c}\text { Power } \\
(W)\end{array}$ & $\begin{array}{c}\text { Fairness } \\
(F I)\end{array}$ & $\begin{array}{c}\text { Time } \\
(s)\end{array}$ \\
\hline \multirow{3}{*}{ Atlanta } & \multirow{3}{*}{15} & \multirow{3}{*}{22} & \multirow{3}{*}{210} & 0.5 & 78.78 & 0 & 960 & 0.68 & 1183.48 \\
\hline & & & & 1 & 78.78 & 0 & 960 & 0.68 & 1637.89 \\
\hline & & & & 2.5 & 75.75 & 7.3 & 762 & 0.61 & 10800 \\
\hline \multirow{3}{*}{ Germany50 } & \multirow{3}{*}{50} & \multirow{3}{*}{88} & \multirow{3}{*}{662} & 0.5 & 72.72 & 32 & 4560 & 0.52 & 10800 \\
\hline & & & & 1 & 71.21 & 35 & 4740 & 0.64 & 10800 \\
\hline & & & & 2.5 & 66.66 & 40 & 5280 & 0.47 & 10800 \\
\hline \multirow{3}{*}{ Nobel-germany } & \multirow{3}{*}{17} & \multirow{3}{*}{26} & \multirow{3}{*}{121} & 0.5 & 79.48 & 0 & 1110 & 0.40 & 1902.95 \\
\hline & & & & 1 & 79.48 & 0 & 1110 & 0.45 & 1460.56 \\
\hline & & & & 2.5 & 76.92 & 0 & 1200 & 0.45 & 6203.29 \\
\hline \multirow{3}{*}{ Polska } & \multirow{3}{*}{12} & \multirow{3}{*}{18} & \multirow{3}{*}{66} & 0.5 & 74.07 & 0 & 900 & 0.76 & 141.7 \\
\hline & & & & 1 & 44.44 & 0 & 1350 & 0.73 & 320.96 \\
\hline & & & & 2.5 & 37.03 & 0 & 1800 & 0.69 & 1736.75 \\
\hline
\end{tabular}

Table III: G-SPB heuristic algorithm

\begin{tabular}{|c|c|c|c|c|c|c|c|c|}
\hline Network & $|V|$ & $|E|$ & $|D|$ & $\begin{array}{c}\text { load } \\
(\gamma)\end{array}$ & $\begin{array}{c}\text { Saving } \\
(\eta \%)\end{array}$ & $\begin{array}{c}\text { Power } \\
(W)\end{array}$ & $\begin{array}{c}\text { Fairness } \\
F I\end{array}$ & $\begin{array}{c}\text { Time } \\
(s)\end{array}$ \\
\hline \multirow{3}{*}{ Atlanta } & \multirow{3}{*}{15} & \multirow{3}{*}{22} & \multirow{3}{*}{210} & 0.5 & 71.21 & 1055 & 0.46 & \multirow{3}{*}{$<84$} \\
\hline & & & & 1 & 68.18 & 1275 & 0.51 & \\
\hline & & & & 2.5 & 51.51 & 1770 & 0.43 & \\
\hline \multirow{3}{*}{ Germany 50} & \multirow{3}{*}{50} & \multirow{3}{*}{88} & \multirow{3}{*}{662} & 0.5 & 81.44 & 3525 & 0.75 & \multirow{3}{*}{$<7000$} \\
\hline & & & & 1 & 81.06 & 3570 & 0.72 & \\
\hline & & & & 2.5 & 66.29 & 5325 & 0.67 & \\
\hline \multirow{3}{*}{ Nobel-germany } & \multirow{3}{*}{17} & \multirow{3}{*}{26} & \multirow{3}{*}{121} & 0.5 & 74.35 & 1290 & 0.51 & \multirow{3}{*}{$<40$} \\
\hline & & & & 1 & 74.35 & 1290 & 0.51 & \\
\hline & & & & 2.5 & 69.23 & 1470 & 0.46 & \\
\hline \multirow{3}{*}{ Polska } & \multirow{3}{*}{12} & \multirow{3}{*}{18} & \multirow{3}{*}{66} & 0.5 & 70.03 & 990 & 0.79 & \multirow{3}{*}{$<20$} \\
\hline & & & & 1 & 70.03 & 990 & 0.79 & \\
\hline & & & & 2.5 & 37.03 & 1800 & 0.76 & \\
\hline
\end{tabular}

Table IV: FG-SPB heuristic algorithm

\begin{tabular}{|c|c|c|c|c|c|c|c|c|}
\hline Network & $|V|$ & $|E|$ & $|D|$ & $\begin{array}{c}\text { load } \\
(\gamma)\end{array}$ & $\begin{array}{c}\text { Saving } \\
(\eta \%)\end{array}$ & $\begin{array}{c}\text { Power } \\
(W)\end{array}$ & $\begin{array}{c}\text { Fairness } \\
F I\end{array}$ & $\begin{array}{c}\text { Time } \\
(s)\end{array}$ \\
\hline \multirow{3}{*}{ Atlanta } & \multirow{3}{*}{15} & \multirow{3}{*}{22} & \multirow{3}{*}{210} & 0.5 & 68.18 & 1275 & 0.51 & \multirow{3}{*}{$<30$} \\
\hline & & & & 1 & 68.18 & 1275 & 0.51 & \\
\hline & & & & 2.5 & 51.51 & 1770 & 0.43 & \\
\hline \multirow{3}{*}{ Germany 50} & \multirow{3}{*}{50} & \multirow{3}{*}{88} & \multirow{3}{*}{662} & 0.5 & 67.04 & 5235 & 0.62 & \multirow{3}{*}{$<5245$} \\
\hline & & & & 1 & 65.90 & 5370 & 0.62 & \\
\hline & & & & 2.5 & 64.77 & 5505 & 0.59 & \\
\hline \multirow{3}{*}{ Nobel-germany } & \multirow{3}{*}{17} & \multirow{3}{*}{26} & \multirow{3}{*}{121} & 0.5 & 74.35 & 1290 & 0.51 & \multirow{3}{*}{$<26$} \\
\hline & & & & 1 & 74.35 & 1290 & 0.51 & \\
\hline & & & & 2.5 & 71.79 & 1380 & 0.46 & \\
\hline \multirow{3}{*}{ Polska } & \multirow{3}{*}{12} & \multirow{3}{*}{18} & \multirow{3}{*}{66} & 0.5 & 70.03 & 990 & 0.79 & \multirow{3}{*}{$<8$} \\
\hline & & & & 1 & 70.03 & 990 & 0.79 & \\
\hline & & & & 2.5 & 68.51 & 1035 & 0.78 & \\
\hline
\end{tabular}

SPB (FG-SPB), which are compliant to Carrier Ethernet network operating with Shortest Path Bridging (SPB) protocol. We formulated the problem as a mixed integer linear program (MILP) that aims at maximizing the number of cables to be powered off while fulfilling the given traffic demand and the ECMP routing rules. Both MILP algorithm and heuristics have been tested on four realistic topologies taking into account three different traffic loads. Experiments prove also that the heuristics are appropriated as energy efficient routing in Carrier Ethernet networks. Based on the obtained encouraging results, our future work will focus on optimizing the initial weight setting based on forecast demands, with the aim to get efficient routing cost.

\section{REFERENCES}

[1] A Kirstädter, C Gruber, J Riedl, and T Bauschert. Carriergrade ethernet for packet core networks. In Asia-Pacific Optical Communications, pages 635414-635414. International Society for Optics and Photonics, 2006.

[2] Rong Fu, Yanmeng Wang, and Michael S Berger. Carrier ethernet network control plane based on the next generation network. In Innovations in NGN: Future Network and Services, 2008. K-INGN 2008. First ITU-T Kaleidoscope Academic Conference, pages 293-298. IEEE, 2008.

[3] Saurabh Mehta, Sandeep Joshi, Tamal Das, and Ashwin Gumaste. On control plane algorithms for carrier ethernet networks: Unicast, multicast provisioning and control traffic 
reduction. Optical Switching and Networking, 23:52-66, 2017.

[4] Yueping Cai, Yao Yan, Zhenghao Zhang, and Yuanyuan Yang. Survey on converged data center networks with dcb and fcoe: standards and protocols. IEEE Network, 27(4):27-32, 2013.

[5] Joseph Chabarek, Joel Sommers, Paul Barford, Cristian Estan, David Tsiang, and Steve Wright. Power awareness in network design and routing. In INFOCOM 2008. The 27th Conference on Computer Communications. IEEE. IEEE, 2008.

[6] JC Cardona Restrepo, Claus G Gruber, and C Mas Machuca. Energy profile aware routing. In 2009 IEEE International Conference on Communications Workshops, pages 1-5. IEEE, 2009.

[7] Priya Mahadevan, Puneet Sharma, Sujata Banerjee, and Parthasarathy Ranganathan. A power benchmarking framework for network devices. In International Conference on Research in Networking, pages 795-808. Springer, 2009.

[8] IEEE standard for local and metropolitan area networksmedia access control (mac) bridges and virtual bridged local area networks-amendment 20: Shortest path bridging. IEEE Std 802.1aq-2012, pages 1-340, 2012.

[9] David Allan, Peter Ashwood-Smith, Nigel Bragg, János Farkas, Don Fedyk, Michel Ouellete, Mick Seaman, and Paul Unbehagen. Shortest path bridging: Efficient control of larger ethernet networks. IEEE Communications Magazine, 48(10):128-135, 2010.

[10] David Allan, Janos Farkas, and Scott Mansfield. Intelligent load balancing for shortest path bridging. IEEE Communications Magazine, 50(7):163-167, 2012.

[11] IEEE standard for local and metropolitan area networks-link aggregation. IEEE Std 802.1AX-2008, pages 1-163, Nov 2008 .

[12] IEEE standard for local and metropolitan area networks - link aggregation. IEEE Std 802.1AX-2014 (Revision of IEEE Std 802.1AX-2008), pages 1-344, Dec 2014.

[13] Joanna Moulierac and Truong Khoa Phan. Optimizing igp link weights for energy-efficiency in multi-period traffic matrices. Computer Communications, 61:79-89, 2015.

[14] Ken Christensen, Pedro Reviriego, Bruce Nordman, Michael Bennett, Mehrgan Mostowfi, and Juan Antonio Maestro. Ieee 802.3 az: the road to energy efficient ethernet. IEEE Communications Magazine, 48(11):50-56, 2010.

[15] Antonio Capone, Daniele Corti, Luca Gianoli, and Brunilde Sansó. An optimization framework for the energy management of carrier ethernet networks with multiple spanning trees. Computer Networks, 56(17):3666-3681, 2012.

[16] Edoardo Amaldi, Antonio Capone, and Luca G Gianoli. Energy-aware ip traffic engineering with shortest path routing. Computer Networks, 57(6):1503-1517, 2013.

[17] Frederic Francois, Ning Wang, Klaus Moessner, Stylianos Georgoulas, and $\mathrm{Ke} \mathrm{Xu}$. Green igp link weights for energyefficiency and load-balancing in ip backbone networks. In IFIP Networking Conference, 2013, pages 1-9. IEEE, 2013.
[18] Luca Chiaraviglio, Marco Mellia, and Fabio Neri. Minimizing isp network energy cost: formulation and solutions. IEEE/ACM Transactions on Networking (TON), 20(2):463476, 2012.

[19] Bernard Fortz and Mikkel Thorup. Internet traffic engineering by optimizing ospf weights. In INFOCOM 2000. Nineteenth annual joint conference of the IEEE computer and communications societies. Proceedings. IEEE, volume 2, pages 519 528. IEEE, 2000.

[20] Will Fisher, Martin Suchara, and Jennifer Rexford. Greening backbone networks: reducing energy consumption by shutting off cables in bundled links. In Proceedings of the first ACM SIGCOMM workshop on Green networking, pages 29-34. ACM, 2010.

[21] Gongqi Lin, Sieteng Soh, and Kwan-Wu Chin. Energyaware traffic engineering with reliability constraint. Computer Communications, 57:115-128, 2015.

[22] Christian Raack, Arie MCA Koster, Sebastian Orlowski, and Roland Wessäly. On cut-based inequalities for capacitated network design polyhedra. Networks, 57(2):141-156, 2011.

[23] Jin Y Yen. Finding the k shortest loopless paths in a network. management Science, 17(11):712-716, 1971.

[24] Sebastian Orlowski, Roland Wessäly, Michal Pióro, and Artur Tomaszewski. Sndlib 1.0 survivable network design library. Networks, 55(3):276-286, 2010.

[25] Per Nikolaj D Bukh and Raj Jain. The art of computer systems performance analysis, techniques for experimental design, measurement, simulation and modeling, 1992.

[26] Concert technology version 12.1. ftp://public.dhe.ibm. com/software/websphere/ilog/docs/optimization/cplex/ refcppcplex.pdf.

[27] Marshall L Fisher. The lagrangian relaxation method for solving integer programming problems. Management science, 27(1):1-18, 1981 . 